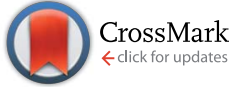

Cite this: RSC Adv., 2017, 7, 12346

Received 28th December 2016 Accepted 15th February 2017

DOI: 10.1039/c6ra28778f

rsc.li/rsc-advances

\section{Microwave-assisted hydrolysis of biomass over activated carbon supported polyoxometalates $\uparrow$}

\author{
S. Tsubaki, ${ }^{\star a}$ K. Oono, ${ }^{\text {b }}$ A. Onda, ${ }^{\text {b }}$ T. Ueda, ${ }^{c}$ T. Mitani ${ }^{d}$ and M. Hiraoka ${ }^{e}$ \\ Activated carbon supported polyoxometalates (AC-POMs) were used for acceleration of hydrolysis of \\ biomass under microwave irradiation. Microwaves exhibited a higher saccharification rate for cellobiose \\ and green seaweed (Monostroma latissimum) over AC-POMs than conductive heating by 1.25 to 1.55 - \\ fold. The activity of the AC-POMs was positively correlated with the dielectric loss of the catalysts, \\ suggesting that microwave susceptibility of AC-POMs is relevant to the enhancement of catalysis under \\ microwave irradiation.
}

Hydrolysis of biomass is one of the key technologies for the production of bio-fuels, bio-materials, and bio-chemicals as well as biologically functional chemicals. ${ }^{1}$ Heterogeneous catalysts have been paid great attention in the green conversion of biomass since they are less hazardous than conventional mineral acids, and do not require neutralization. ${ }^{2}$ For instance, sulfonated carbons effectively hydrolyze crystalline cellulose as water-tolerant solid acids. ${ }^{3}$

Microwave irradiation is widely used for accelerating organic and inorganic reactions, ${ }^{4}$ materials processing, ${ }^{5}$ environmental treatment ${ }^{6}$ and biomass treatment. ${ }^{7}$ Microwave irradiation is capable of selectively heating microwave sensitizing materials by dielectric and conduction loss mechanisms. ${ }^{8}$ Solid catalysts such as carbon supported Pt and carbon-filled zeolite can be selectively heated in less microwave susceptive reaction media to enhance heterogeneous reactions by generating local hot spots in the vicinity of the solid catalysts. ${ }^{9}$ A combination of microwaves and a heterogeneous catalyst is, therefore, expected to be effective for enhancing biomass conversion processes. ${ }^{10}$

Polyoxometalates (POMs) are well-known for super-strong acid and oxidation activities with less corrosivity than mineral acids. ${ }^{11}$ POMs have been applied for hydrolysis of crystalline cellulose ${ }^{12}$ delignification of lignocellulosic biomass for paper

\footnotetext{
${ }^{a}$ Department of Chemical Science and Engineering, School of Materials and Chemical Technology, Tokyo Institute of Technology, 2-12-1, Ookayama, Meguro, Tokyo, 152-8550, Japan. E-mail: shuntaro.tsubaki@gmail.com; tsubaki.s.aa@m.titech.ac.jp ${ }^{b}$ Research Laboratory of Hydrothermal Chemistry, Faculty of Science, Kochi University, Akebono-cho 2-5-1, Kochi 780-8520, Japan

'Department of Marine Resource Science, Faculty of Agriculture and Marine Sciences, Kochi University, Akebono-cho 2-5-1, Kochi, Japan

${ }^{d}$ Research Institute for Sustainable Humanosphere, Kyoto University, Gokasho, Uji, 611-0011, Japan

${ }^{e}$ Usa Marine Biological Institute, Kochi University, Inoshiri, Usa, Tosa, Kochi 7811164, Japan

$\dagger$ Electronic supplementary information (ESI) available. See DOI: 10.1039/c6ra28778f
}

industry, ${ }^{13}$ oxidation of cellulose and hemicellulose ${ }^{14}$ and dehydration of hexose to 5-hydroxymethyl furfural. ${ }^{15}$ Previously, we have reported that homogeneous POMs facilitate hydrolysis of polysaccharides (starch and crystalline cellulose) and green seaweed (Ulva spp.) under microwaves. ${ }^{16}$ POMs exhibited higher activity than conventionally used mineral acids such as $\mathrm{HCl}$ and $\mathrm{H}_{2} \mathrm{SO}_{4}$. For greener reaction, however, heterogeneous POMs are preferred for easier separation and recyclability.

Solid POMs such as $\mathrm{Cs}^{+}$salts and supported POMs have been used for catalyst, energy storage and sensing applications. ${ }^{11,17}$ Activated carbon (AC) is one of the useful catalyst support as well as microwave susceptors. ${ }^{9}$ Previously, we have reported that several types of ACs were effective for hydrolysis of corn starch with reduction in the reaction temperature..$^{18}$ In the present study, microwave-susceptible solid acid catalysts were prepared by supporting POMs on ACs and used to study the effects of microwave generated local hot spot at the solid acid catalyst.

Microwave-susceptibility of susceptors were first compared among AC (type A, Table 1), silicon carbide (SiC) and yttria stabilized zirconia (YSZ) which are typically used microwave susceptors. These susceptors $(500 \mathrm{mg}$ ) were suspended in water $(10 \mathrm{~mL})$ and microwaved by multimode microwave reactor START-D (2.45 GHz, Milestone, Inc.) equipped with PTFE highpressure reactor and PID temperature control connected to the thermocouple thermometer. Microwave susceptibility of the supports were evaluated by the microwave energy consumption monitored by the START-D controller. ACs exhibited the best microwave susceptibility and reduced microwave energy consumption down by $39 \%$ from that of pure water (Fig. 1).

Silicotungstate (SiW) was, then, supported on 7 kinds of ACs (Table 1) by the wet impregnation method according to Kumar et al. ${ }^{19}$ Namely, ACs were suspended in $50 \mathrm{mM}$ methanol solution of SiW and shaken overnight, then, methanol was removed by evaporation. Where indicated, ACs were pretreated with 1 wt $\%$ aqueous solutions of $\mathrm{HCl}, \mathrm{LiCl}, \mathrm{NaCl}, \mathrm{CsCl}, \mathrm{KCl}$, tetrabutyl ammonium bromide (TBABr), $\mathrm{NH}_{4} \mathrm{Cl}$, and aqueous $\mathrm{HNO}_{3}$ 
Table 1 Descriptions and supplier of ACs used in this study

\begin{tabular}{lll}
\hline Type & Description of ACs & Supplier \\
\hline A & $\begin{array}{l}\text { Fracture type } \\
\text { activated carbon }\end{array}$ & $\begin{array}{l}\text { Wako Pure Chemical } \\
\text { Industries Ltd. }\end{array}$ \\
B & Bone char & \\
C & SX II & \\
D & SX PLUS & Ajinomoto Fine-Techno Co., Inc. \\
E & SD & Osaka Gas Chemicals Co., Ltd. \\
F & SHIRASAGI \\
G & SHIRASAGI & \\
& &
\end{tabular}

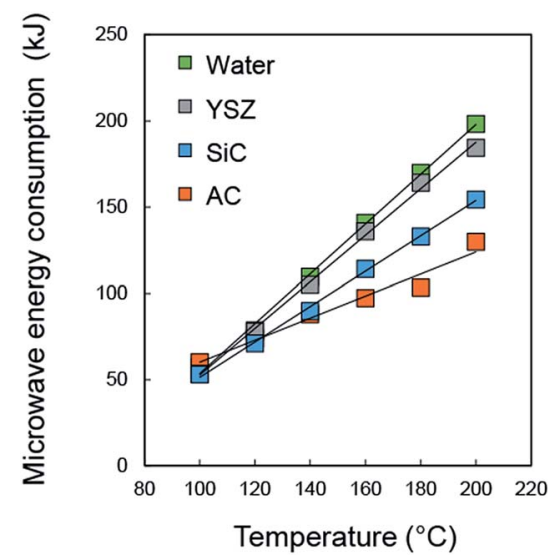

Fig. 1 Comparison of microwave energy consumption of aqueous suspension of $\mathrm{AC}$ (type $\mathrm{A}$ ), $\mathrm{SiC}$ and YSZ under hydrothermal condition $\left(100-200^{\circ} \mathrm{C}\right)$.

solutions (10\% and $20 \%$ ) overnight to stabilize POM anions on ACs. The pretreatment solutions were thoroughly washed with water before supporting SiWs.

The aqueous suspension of POM-impregnated ACs were, then, repeatedly washed under hydrothermal condition using microwaves (START-D microwave oven, $200{ }^{\circ} \mathrm{C}$ for $10 \mathrm{~min}$ ) to remove loosely adsorbed POMs. Subsequently, they were thoroughly washed with water and dried at $250{ }^{\circ} \mathrm{C}$ for 6 hours to obtain AC-SiW. The amount of SiW supported on ACs were also described as a function of size and BET specific surface area (Fig. 2). ACs larger than $40 \mu \mathrm{m} \times 20 \mu \mathrm{m}$ (types $\mathrm{D}-\mathrm{G}$ ) were found effective for supporting SiW. Similarly, the BET specific surface area and micropore volume above $800 \mathrm{~m}^{2} \mathrm{~g}^{-1}$ and $1200 \mathrm{~m}^{2} \mathrm{~g}^{-1}$ were sufficient for supporting SiW, respectively.

Several kinds of AC-SiW were further prepared by using types D, E, F with several pretreatments (Table 2). AC-SiWs contained $2.79 \times 10^{-5} \mathrm{~mol} \mathrm{~g}^{-1}$ to $5.12 \times 10^{-5} \mathrm{~mol} \mathrm{~g}^{-1}$. The amounts of acid sites of AC-POMs were, then, determined by the titration method. Namely, the AC-POMs were suspended in $10 \mathrm{mM}$ $\mathrm{NaOH}$ and stirred for 2 hours. The AC-POMs were removed by centrifugation. The supernatant $(10 \mathrm{~mL})$ was titrated with $10 \mathrm{mM} \mathrm{HCl}$. The amounts of acid sites of AC-SiWs attained 5.00 $\times 10^{-4}$ to $3.40 \times 10^{-3} \mathrm{~mol} \mathrm{~g}^{-1}$ as $\mathrm{HCl}$ equivalent (Table 2 ).

Cellobiose was used as model biomass substrate for testing catalysis of AC-SiWs. Cellobiose (100 mg) were suspended in pure water $(10 \mathrm{~mL})$ with AC-POM $(100 \mathrm{mg})$ and hydrolyzed
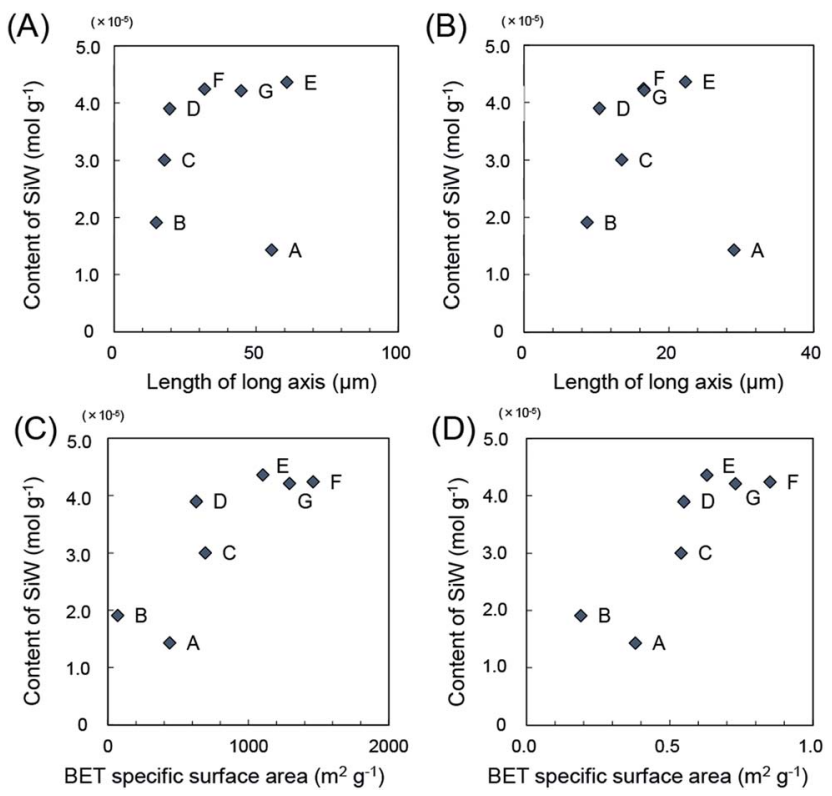

Fig. 2 The amount of SiW supported on ACs as functions of (A and B) size of $A C s$ and (C and D) BET nitrogen adsorption properties. Alphabets in the figures show the types of ACs as shown in Table 1.

Table 2 The amount of SiW and their acid sites

\begin{tabular}{lll}
\hline $\begin{array}{l}\text { POM-pretreatment } \\
\text { (type of AC) }\end{array}$ & $\begin{array}{l}\text { Amount of SiW } \\
\left(\mathrm{mol} \mathrm{g}^{-1}\right)\end{array}$ & $\begin{array}{l}\text { Amount of acid } \\
\text { sites }^{a}\left(\mathrm{~mol} \mathrm{~g}^{-1}\right)\end{array}$ \\
\hline SiW-none (type D) & $3.90 \times 10^{-5}$ & $5.00 \times 10^{-4}$ \\
SiW-HCl (type D) & $4.52 \times 10^{-5}$ & $1.82 \times 10^{-3}$ \\
SiW-LiCl (type D) & $4.92 \times 10^{-5}$ & $1.19 \times 10^{-3}$ \\
SiW-NaCl (type D) & $4.49 \times 10^{-5}$ & $1.90 \times 10^{-3}$ \\
SiW-KCl (type D) & $5.12 \times 10^{-5}$ & $2.13 \times 10^{-3}$ \\
SiW-CsCl (type D) & $3.77 \times 10^{-5}$ & $2.07 \times 10^{-3}$ \\
SiW-TBABr (type D) & $4.70 \times 10^{-5}$ & $2.23 \times 10^{-3}$ \\
SiW-NH 4 Cl (type D) & $3.66 \times 10^{-5}$ & $2.38 \times 10^{-3}$ \\
SiW-HNO $10 \%$ (type D) & $3.69 \times 10^{-5}$ & $1.61 \times 10^{-3}$ \\
SiW-HNO 3 20\% (type D) & $2.79 \times 10^{-5}$ & $1.74 \times 10^{-3}$ \\
SiW-none (type E) & $4.24 \times 10^{-5}$ & $3.09 \times 10^{-3}$ \\
SiW-HCl (type E) $^{-5}$ & $4.78 \times 10^{-5}$ & $3.08 \times 10^{-3}$ \\
SiW-none (type F) & $4.21 \times 10^{-5}$ & $3.40 \times 10^{-3}$ \\
${ }^{a}$ HCl equivalent acid sites. & & \\
\end{tabular}

under microwaves using START-D as mentioned above. The microwave reaction was operated at $180{ }^{\circ} \mathrm{C}$ for $10 \mathrm{~min}$ with $8 \mathrm{~min}$ of heating-up time. The generation of glucose was monitored by HPLC (LC-2000 system, JASCO) equipped with a column of Shodex SC1011 $(7.8 \mathrm{~mm}$ I.D $\times 300 \mathrm{~mm}$, Showa Denko K.K.) with eluent of water at $1 \mathrm{~mL} \mathrm{~min}^{-1}$ of flow rate and RI detection. Saccharification rate was calculated as a follows;

$$
\text { Saccharification rate }(\%)=100 \times \frac{0.95 \times \text { glucose yield }}{\text { initial cellobiose load }}
$$

The amount of SiW leaching was monitored by ICP-OES. The saccharification rate attained $30.7 \%$ to $54.7 \%$ depending on the pretreatment of the catalyst (Table 3 ). The amounts of leached 
Table 3 The amount of SiW and their acid sites

\begin{tabular}{|c|c|c|}
\hline $\begin{array}{l}\text { POM-pretreatment } \\
\text { (type of AC) }\end{array}$ & $\begin{array}{l}\text { Saccharification } \\
\text { rate }(\%)\end{array}$ & $\begin{array}{l}\text { Amount of } \\
\text { leached SiW }(\mu \mathrm{M})\end{array}$ \\
\hline SiW-none (type D) & 40.7 & 6.4 \\
\hline SiW-HCl (type D) & 43.3 & 6.3 \\
\hline SiW-LiCl (type D) & 39.8 & 5.0 \\
\hline SiW-NaCl (type D) & 35.7 & 5.6 \\
\hline SiW-KCl (type D) & 38.8 & 6.1 \\
\hline SiW-CsCl (type D) & 34.2 & 5.0 \\
\hline SiW-TBAB (type D) & 35.3 & 4.0 \\
\hline SiW- $\mathrm{NH}_{4} \mathrm{Cl}$ (type D) & 37.4 & 5.2 \\
\hline SiW- $\mathrm{HNO}_{3} 10 \%$ (type D) & 35.1 & 12 \\
\hline $\mathrm{SiW}-\mathrm{HNO}_{3} 20 \%$ (type D) & 30.7 & 16 \\
\hline SiW-none (type E) & 46.5 & 12 \\
\hline SiW-HCl (type E) & 54.7 & 8.0 \\
\hline SiW-none (type F) & 44.4 & 14 \\
\hline
\end{tabular}

SiW were maintained below $10 \mu \mathrm{M}$, however, oxidation of AC by $\mathrm{HNO}_{3}$ gave higher SiW leaching than other pretreatments (Table 3). The amount of leached SiW attained 4.6\% (SiW-LiCl) to $22 \%\left(\mathrm{SiW}-\mathrm{HNO}_{3} 10 \%\right.$ and $\mathrm{SiW}-\mathrm{HNO}_{3} 20 \%$ ) of the immobilized SiW (Table $\mathrm{S} 1 \dagger$ ).

The activities of AC-SiWs were, then, compared under microwave and conductive heating under the same thermal history using induction oven (SSN-400, Shikoku Rika. Co., Kochi, Japan). ${ }^{16}$ Microwaves gave higher saccharification rate

\section{(A) Cellobiose (AC-SiW)}

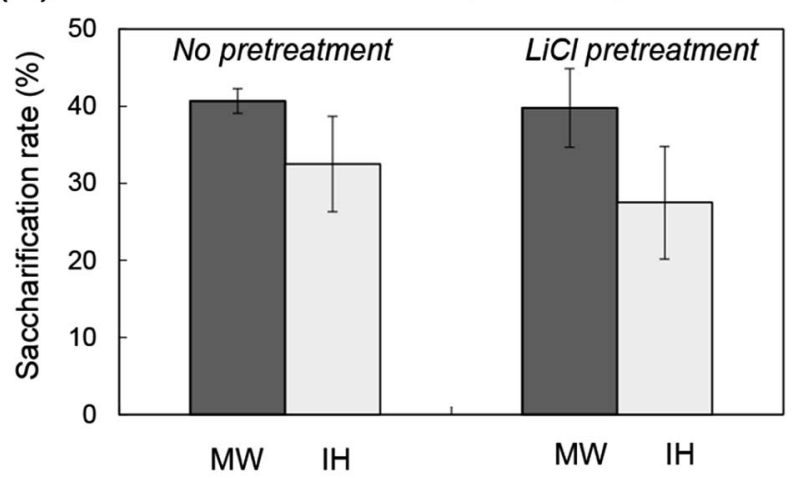

(B)

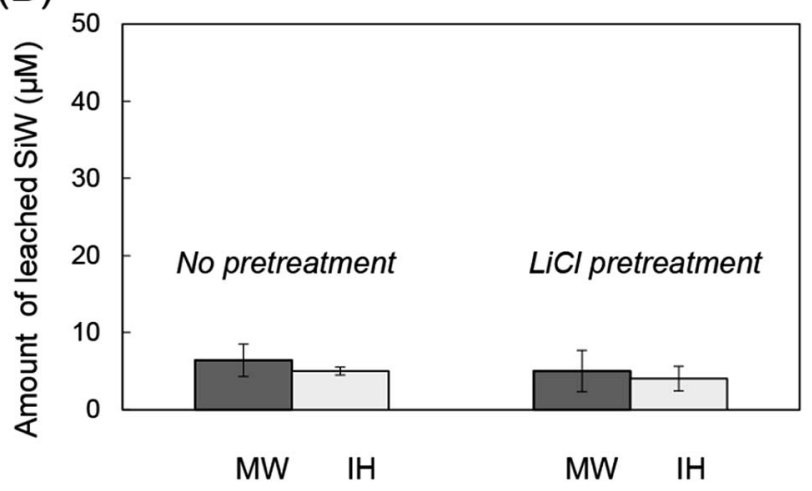

Fig. 3 Comparison of microwave $(\mathrm{MW})$ and induction heating $(\mathrm{IH})$ for hydrolysis of cellobiose over AC-SiW. (A) Saccharification rate of cellobiose and (B) amount of leached SiW during reaction. than induction heating by 1.25 - to 1.45 -fold without change in the amount of leached SiW during reaction (Fig. 3).

In addition, the effects of AC-supported phosphotungstic acid (AC-PW) were tested for hydrolysis of green seaweed biomass (Monostroma latissimum). AC-PW was prepared by the same procedure for AC-SiW using type A activated carbon without any pretreatment. Dried and powdered M. latissimum (500 mg) and AC-PW (500 mg) were suspended in distilled water $(10 \mathrm{~mL})$ and microwaved at $140{ }^{\circ} \mathrm{C}$ and $10 \mathrm{~min}$. The amount of generated total sugar (including both native polysaccharide and hydrolyzed sugars) were determined by the phenol sulfuric acid. The amount of reducing sugars generated after hydrolysis were determined by the DNS method.

Hydrolysis of $M$. latissimum over AC-PW produced 400$420 \mathrm{mg}$ of total sugars by both microwave and induction heating (Fig. 4A). Microwaves, however, exhibited higher yield in reducing sugar than the induction heating by 1.55-fold (Fig. 4B) with no difference in PW leaching (Fig. 4C). The results indicated that hydrolysis of $M$. latissimum was enhanced under microwaves to produce lower molecular reducing sugars such as rhamnan sulfate oligosaccharides. The hydrolyzed polysaccharide (rhaman sulfate) of $M$. latissimum are expected for their biological effects such as anticoagulant activity and antiherpetic effect. ${ }^{20}$

These results demonstrated that AC-POMs exhibit higher saccharification of carbohydrates under microwaves than conductive heating. Direct heating of AC-POM catalysts due to microwaves probably generated local hot spot at the solid catalyst to enhance hydrolysis of biomass. To understand the mechanism of energy transfer from microwaves to AC-POM, their complex dielectric constants in water were further examined using the coaxial probe method in the same concentration

\section{Monostroma latissimum (AC-PW)}

(A)

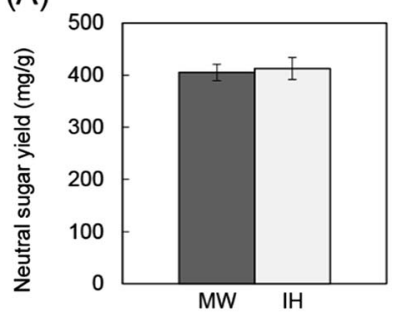

(B)

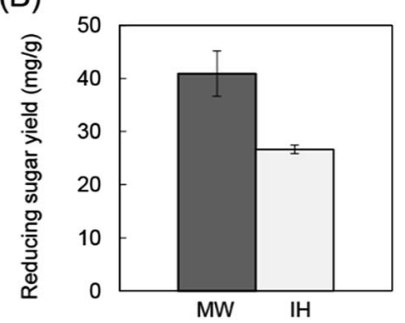

(C)

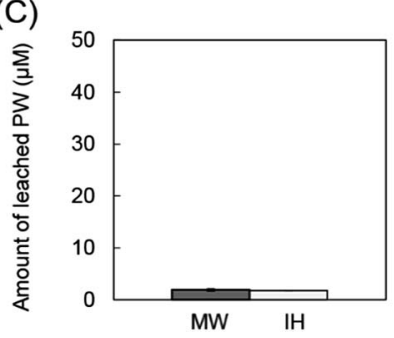

Fig. 4 Comparison of microwave (MW) and induction heating (IH) for hydrolysis of green seaweed (Monostroma latissimum) over AC-PW. (A) Neutral sugar yield, (B) reducing sugar yield and (C) amount of leached PW during reaction. 
as the cellobiose hydrolysis (100 $\mathrm{mg}$ in $10 \mathrm{~mL}$ ). Dielectric loss tangent $(\tan \delta)$ at $2.45 \mathrm{GHz}$ was calculated from the dielectric constant using the following equation;

$$
\tan \delta=\frac{\varepsilon^{\prime \prime}}{\varepsilon^{\prime}}
$$

where, $\varepsilon^{\prime}$ and $\varepsilon^{\prime \prime}$ are relative permittivity and dielectric loss at $2.45 \mathrm{GHz}$, respectively. The saccharification rate of cellobiose over AC-SiW were, then, plotted over relative permittivity $\left(\varepsilon^{\prime}\right)$, dielectric loss $\left(\varepsilon^{\prime \prime}\right)$, dielectric loss tangent $(\tan \delta)$, acid sites as well as the amount of leached SiW (Fig. 4).

Relative permittivity, dielectric loss and dielectric loss tangent exhibited positive correlations with cellobiose saccharification indicating that complex dielectric properties of AC-POMs are one of the key parameter for catalysis under microwaves (Fig. 5A-C). Stronger microwave absorption by ACSiWs can generate higher temperature at the catalyst. We assume that higher saccharification rate can be obtained due to the higher local hot spot generated by stronger microwave absorption of the catalyst. Another key parameter is the acidity of the AC-SiWs. The increase in the amount of acid sites also facilitated saccharification of cellobiose (Fig. 5D) without
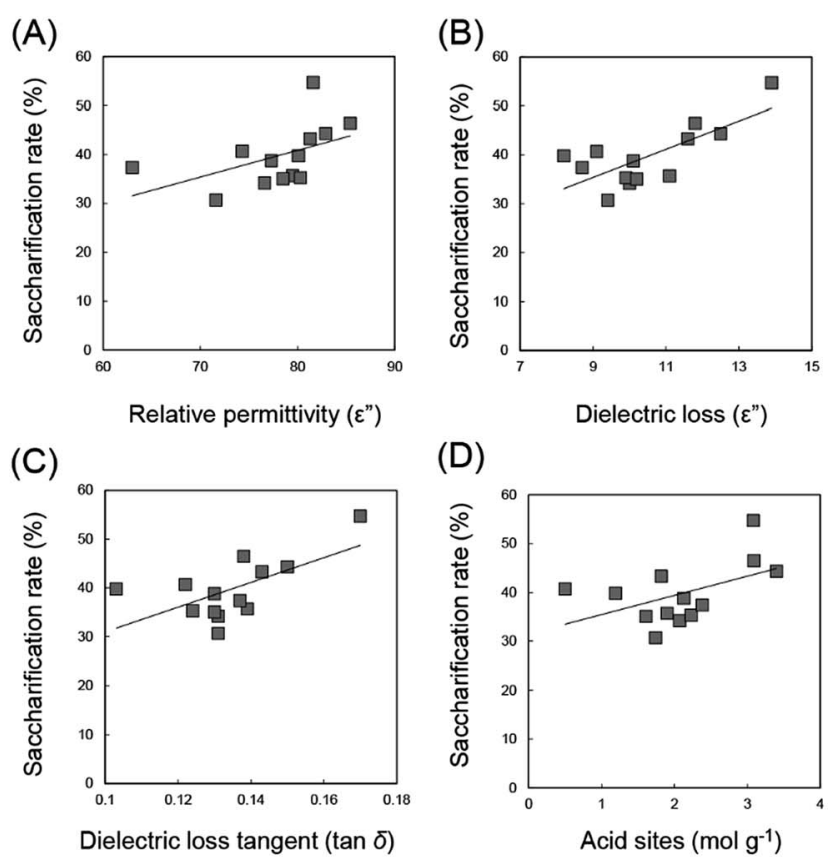

(D)

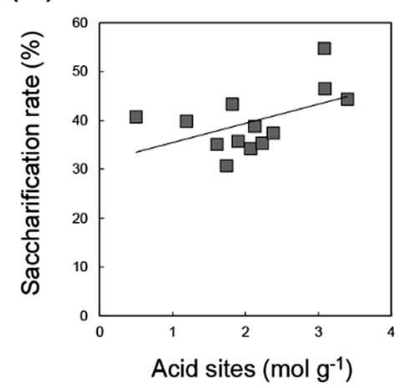

(E)

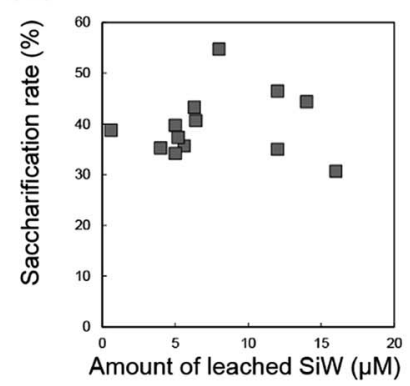

(F)

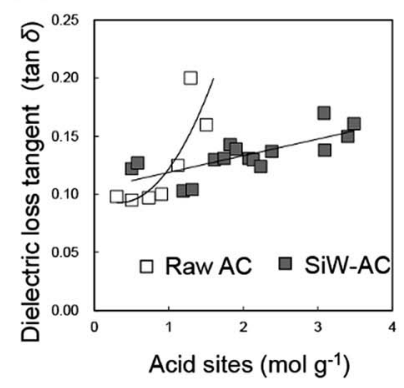

Fig. 5 Correlations among dielectric properties of AC-SiW, saccharification rate of cellobiose, acid sites and the amount of leached POM. significant correlation with the amount of leached SiW (Fig. 5E).

In addition, the dielectric loss tangent of AC-SiW positively correlated with the amount of acid sites (Fig. 5F). This could be due to enhanced dielectric loss due to ionic conduction of protons at the acid sites. The ionic conduction of electrolytes are one of the important microwave absorption mechanisms for heating materials. ${ }^{8}$ Because protons exhibit significantly higher ion conductivity than other ions by the Grotthuss mechanism, ${ }^{21}$ the ionic conduction of proton at the acid sites can improve the dielectric loss of the catalyst. The same phenomenon was also observed for raw ACs depending on the amount of acidic functional groups, such as hydroxyl and carboxyl groups, originally existing on the carbon surface (Fig. 5F). The microwave susceptibility of AC-POMs was, therefore, originated from the synergy of dielectric loss of AC support as well as ionic conduction of protons of the acid sites.

\section{Conclusions}

In this study, the effects of microwave local heating were investigated on hydrolysis of biomass using solid POMs. AC supports were used since they exhibit high microwave susceptibility. AC-POMs were, then, fabricated by the wet impregnation method. Microwaves exhibited higher saccharification rate of cellobiose over AC-SiW than induction heating by 1.25- to 1.45-fold. AC-PW also generated higher reducing sugars under microwave than induction heating by 1.55 -fold. Positive correlation was observed between dielectric loss of AC-SiW and saccharification rate. The results indicated that direct and selective heating of AC-POMs facilitates hydrolysis of biomass under microwaves by generating local hot spot at the solid catalyst.

\section{Acknowledgements}

This study was supported by the Adaptable and Seamless Technology Transfer Program (A-STEP) through Target-driven R\&D (Exploratory Research), Japan Science and Technology Agency (AS242Z03337N), Grant-in-Aid for Young Scientists B Japan Society for the Promotion of Science (15K18814) and the Japan Prize Foundation. The dielectric measurements were conducted through Analysis and Development System for Advanced Materials (ADAM) at the Research Institute for Sustainable Humanosphere, Kyoto University.

\section{Notes and references}

1 A. Sun and J. Cheng, Bioresour. Technol., 2002, 83, 1.

2 R. Rinaldi and F. Schüth, Energy Environ. Sci., 2009, 2, 610.

3 M. Hara, T. Yoshida, A. Takata, J. N. Konda, S. Hayashi and L. Domen, Angew. Chem., Int. Ed., 2004, 43, 2955; A. Onda, T. Ochi and K. Yanagisawa, Green Chem., 2008, 10, 1033.

4 A. Loupy, Microwaves in organic synthesis, Wiley-VCH Verlag GmbH \& Co., KGaA, Weinheim. 2006.

5 Y. V. Bykov, K. I. Rybakov and V. E. Semenov, J. Phys. D: Appl. Phys., 2001, 34, R55. 
6 D. A. Jones, T. P. Lelyveld, S. D. Mavrofidis, S. W. Kingman and N. J. Miles, Resour., Conserv. Recycl., 2002, 34, 75.

7 S. Tsubaki, T. Ueda and A. Onda, in Microwaves in Catalysis, Wiley-VCH Verlag GmbH \& Co., KGaA, Weinheim, 2015.

8 C. Gabriel, S. Gabriel, E. H. Grant, B. S. J. Halstead and D. M. P. Mingos, Chem. Soc. Rev., 1998, 27, 213.

9 S. Horikoshi and N. Serpone, Catal. Sci. Technol., 2014, 4, 1197; D. Mochizuki, R. Sasaki, M. Maitani, M. Okamoto, E. Suzuki and Y. Wada, J. Catal., 2015, 323, 1.

10 Y. B. Huang and Y. Fu, Green Chem., 2013, 15, 1095.

11 I. V. Kozhevnikov, Chem. Rev., 1998, 98, 171.

12 K. I. Shimizu, H. Furukawa, N. Kobayashi, Y. Itaya and A. Satsuma, Green Chem., 2009, 11, 1627.

13 I. A. Weinstock, R. H. Attala, R. S. Reiner, M. A. Moen, K. E. Hammel, C. J. Houtman, C. L. Hill and M. K. Harrup, J. Mol. Catal. A: Chem., 1997, 116, 59.

14 B. B. Sarma and R. Neumann, Nat. Commun., 2014, 5, 4621.
15 C. Fan, H. Guan, H. Zhang, J. Wang, S. Wang and X. Wang, Biomass Bioenergy., 2011, 35, 2659.

16 S. Tsubaki, K. Oono, T. Ueda, A. Onda, K. Yanagisawa, T. Mitani and J. Azuma, Bioresour. Technol., 2013, 144, 67; S. Tsubaki, K. Oono, M. Hiraoka, T. Ueda, A. Onda and K. Yanaigsawa, Green Chem., 2014, 16, 2227.

17 T. Okuhara, Chem. Rev., 2002, 102, 3641; Y. Wang and I. A. Weinstock, Chem. Soc. Rev., 2012, 41, 7479; Y. Ji, L. Huang, J. Hu, C. Streb and Y.-F. Song, Energy Environ. Sci., 2015, 8, 776.

18 A. Matsumoto, S. Tsubaki, M. Sakamoto and J. Azuma, Bioresour. Technol., 2011, 102, 3985.

19 V. B. Kumar, I. N. Pulidinim and A. Gedanken, Renewable Energy, 2015, 78, 141.

20 S. L. Holdt and S. Kraan, J. Appl. Phycol., 2011, 23, 543-597. 21 S. Tsubaki, M. Hiraoka, S. Hadano, K. Okamura, T. Ueda, H. Nishimura, K. Kashimura and T. Mitani, Carbohydr. Polym., 2015, 115, 78. 